\title{
INTRACRANIAL HEMORRHAGE DETECTION IN CT SCAN USING DEEP LEARNING
}

\section{Anas Tharek ${ }^{1}$, Ahmad Sobri Muda ${ }^{1, *}$ Aqilah Baseri Hudi ${ }^{2}$, and Azzam Baseri Hudin ${ }^{3}$}

${ }^{1}$ Universiti Putra Malaysia, Serdang, 43400, Selangor, Malaysia.

2,3 Universiti Kebangsaan Malaysia, Bangi, 43600, Selangor, Malaysia.

*Corresponding Author's Email: asobri@upm.edu.my

Article History: Received December 2, 2021; Revised January 9, 2022;

Accepted January 9, 2022

\begin{abstract}
Missed detection of intracranial hemorrhage in Head CT scans has significantly impacted patient morbidity and mortality. Early detection of intracranial hemorrhage enables patients to receive appropriate treatment which resulted in a better outcome. Some doctors have limited experience in interpreting the $\mathrm{CT}$ scan hence increasing the probability to miss the hemorrhage. The main objective of this study is to develop an algorithm model capable of detecting intracranial hemorrhage in a head CT scan. We are using deep learning from a convolutional neural network (CNN) to produce this algorithm module. This was a cross-sectional study using secondary data, in which 200 data were collected from public datasets. All of the samples have been anonymized into secondary data. The algorithm model is trained using deep learning via a Jupyter Notebook platform. To analyze the algorithm model performance, a confusion matrix was used to measure the accuracy, sensitivity, specificity, precision, and F1 score. This study showed that from 200 training data, 95 samples were true positive, 95 samples were true negative, 7 samples were false positive, and 3 samples were false negative. This algorithm model shows high sensitivity (0.9694), high specificity (0.9314), high precision (0.9314), and high accuracy (0.9500) with an F1 score of 0.9500. This study has proven that deep learning by using CNN enables us to create an accurate classifier that can differentiate between head CT scan with intracranial hemorrhage and without hemorrhage.
\end{abstract}

KEYWORDS: Artificial intelligence, deep learning, convolutional neural network, intracranial hemorrhage, CT Brain. 


\subsection{INTRODUCTION}

Intracranial hemorrhage is a potentially life-threatening neurological condition. It results in a significant burden on health resources. It can happen in many different causes such as due to increased blood pressure, hemorrhage secondary to infarct, trauma, tumor hemorrhage, and many more. One of the common causes of intracranial hemorrhage is traumatic brain injury[1]. When the blood from trauma is in contact with adjacent brain tissues, it irritates and causes swelling. This is known as cerebral edema. The pool of blood within the brain parenchyma is called a hematoma. This causes increased pressure on the adjacent brain tissues which leads to reduced blood flow and kills the brain cells [2].

There are four types of intracranial hemorrhage in trauma characterized by the extravascular accumulation of blood within different intracranial spaces. There are extradural hemorrhage $(\mathrm{EDH})$, subdural hemorrhage (SDH), subarachnoid hemorrhage (SAH), and contusional hemorrhage [3]. Extradural hemorrhage is also known as epidural hemorrhage which is a collection of blood in between the inner surface of the skull and the outer surface of the dura. This is usually associated with a skull fracture. The Source of bleeding is usually from the artery, most commonly the middle meningeal artery. Subdural hemorrhage is a collection of blood within the subdural spaces which is the potential space between the dura and arachnoid of meninges around the brain. Prognosis varies widely depending on the size and chronicity of the hemorrhage. Subarachnoid hemorrhage (SAH) is a type of extra-axial intracranial hemorrhage located within the subarachnoid spaces. Besides trauma, other causes that can lead to subarachnoid hemorrhage are ruptured aneurysm, venous infarction, cerebral vasculitis, and dural arteriovenous malformation. Contusional hemorrhage is a type of intra-axial intracranial hemorrhage which is commonly seen in the setting of significant head injury. It is characterized by foci of hemorrhage in the brain parenchymal which frequently happens in the frontal lobes.

When a patient comes to the hospital after trauma. The emergency department will assess the patient. The detailed neurological examination must be completed after primary and secondary surveys were done. Not all trauma cases need a CT Scan due to radiation risk. NICE (national institute for health and care excellence) has made a guideline to choose which patient requires a CT scan done as the benefit outweighs the risk of radiation [4]. 
Once the CT scan is done, the radiology doctor will review the case. In normal situations, not all cases are being reviewed at the same time the scan was done as they need to handle other modalities in the radiology department. The primary team is the one who usually sees the scan first. In some of the cases, subtle intracranial hemorrhage may be missed by them. This is due to the highly variable appearance of intracranial hemorrhage, depending on its age and location. The most common types of intracranial hemorrhage that were missed were subdural and subarachnoid hemorrhage occurring in 39\% and 33\% of the cases, respectively. The most common location for missed subdural hemorrhage was either parafalcine or frontal [5].

Rapid diagnosis and management of patients with intracranial hemorrhage are important because early deterioration is common in the first few hours after intracranial hemorrhage onsets. More than 20\% of patients will experience a decrease in the Glasgow Coma Scale (GCS) of 2 or more points between the prehospital emergency medical services (EMS) assessment and the initial evaluation in the emergency department (ED) [6]. It is critical for deciding on the need and approach for emergent surgical intervention. The surgical intervention aims to prevent herniation, reduce the intracranial pressure and reduce the pathophysiological impact of the hematoma on the surrounding tissue. This is achieved by reducing the mass effect cellular toxicity of the blood product [7]. Recognizing intracranial hemorrhage in head CT is also essential for allowing safe administration of thrombolytic therapy in acute ischemic stroke. Since "time is brain", increased speed and reduced error in these clinical settings would constitute life-saving innovations.

Machine learning has become popular over the past few years, particularly deep learning which is a branch of machine learning that employs multi-layered neural networks. It has shown huge potential in extracting important information from medical images. Deep learning has been involved in a few medical fields which include grading of detection of metastases in histologic sections of lymph nodes [8], classification of images of skin cancer [9], and diabetic retinopathy on retinal fundus photographs[10] with high accuracies. It has proved to be accurate in image classification and processing tasks, mainly using convolutional neural networks (CNN) [11]. The same method will be implemented in the head CT scan image. It will involve image segmentation and the processed image will be trained by a deep learning approach to detect hemorrhage in the scan which is classified into cases with hemorrhage and cases without hemorrhage with great 
accuracy.

Deep learning transforms the dataset by mapping them with high dimension space. For image classification, the convolutional neural network in deep learning will provide huge support with advanced techniques [12]. CNN extracts the semantic features and network-fused features of the dataset to classify the images [13]. Due to the deep nature of the network, high-resolution image classification is well supported by the convolutional neural network. This is important as most of the medical data must be in high-quality resolution to prevent missed diagnosis of diseases that could harm the patient as this will delay the management [14].

\subsection{METHOD}

\subsection{Study Design}

This was a cross-sectional study using secondary data, in which the 200 data was collected from public datasets. This dataset is owned by Abdul Kader Helwan, academic staff at Al-Manar University of Tripoli, Lebanon [15]. Permission to use the dataset for this research was officially obtained from the owner. All of the samples had been anonymized into secondary data.

The study population consisted of all adult patients who underwent head CT scan with intracranial hemorrhage and without intracranial hemorrhage from public datasets. Inclusion criteria include both gender, CT brain of all types of ICH, CT brain of normal findings, CT of ICH with a formal report which is categorized by the dataset provider. The exclusion criteria include CT brain without anonymization into secondary data, CT brain with artifacts, lowquality CT brain images which is not suitable for making a diagnosis.

The CT brain with radiological findings of intracranial hemorrhage and without intracranial hemorrhage was classified by the dataset provider. A total of $100 \mathrm{CT}$ brain with hemorrhage and another $100 \mathrm{CT}$ brain without hemorrhage was put in two different folders. The dataset was classified based on a formal report of the CT scan.

\subsection{Sampling Method}

The sampling method is simple random sampling. The units in the sampling frame were numbered from 1 to N. A series of random numbers between 1 to $\mathrm{N}$ were drawn by using the Table of Random Number. The units that were selected were the sample. The sample was coded with 1 for cases with intracranial hemorrhage and 0 for cases 
without hemorrhage. A complete list sample of head CT scans was obtained from public datasets. The samples were chosen from the list using a simple random sampling method. All of the samples has been anonymized into secondary data. . The data is in DICOM format. All of this data is converted to PNG format. PNG format is more ready to be used and utilized by any machine learning framework [16].

For the development of the algorithm, we used a Jupyter Notebook as a platform for the training process. Jupyter Notebook is a free and open-source platform with an interactive web tool [17]. It is a webbased application where the system can run the phyton programming language. Python is the most popular language for developing an algorithm for artificial intelligence [18].

\subsection{Convolutional Neural Networks (CNNs)}

There are several types of deep neural networks, one of them is Convolutional Neural Networks (CNNs), which are used to build the algorithm model in this study. A CNN is a type of Feed-Forward Neural Network (FFNN) that is inspired by sophisticated biological processes [19]. It consists of many layers that can be classified into two main types: convolution layer and pooling (sub-sampling) layer. These two types of layers are alternately arranged to form a deep structure. The last layers are fully connected layers that have full connections to the previous layers, as commonly seen in traditional ANNs.

The architecture of the convolutional neural network is as shown in Figure 1. It consists of convolution layers, pooling layers, and fully connected layers. In the first layer, the convolutional filter was used to generate the convolution or feature maps of size by sliding the filter over the image and convolving it with the square input data that fits the kernel. The intracranial hemorrhage features are extracted from input images in the convolution layers. The mathematical operation of convolution preserves the spatial relationship between pixels.

The following layer is called pooling in which the dimensionality of feature maps generated in the first layer is reduced. This operation requires a selection window size from each feature map and takes the largest element from the rectified feature map within that window. Although we may take the average (Average Pooling) or sum of all elements in that selected window, taking the maximum (Max Pooling) has been more common and shown to work better [20]. The pooling operation essentially reduces the spatial dimension of input maps and allows the $\mathrm{CNN}$ to learn some invariance to moderate distortions in the 
training; this feature enhances the generalization of the $\mathrm{CNN}$ at test time as the model is more tolerant to moderate distortion in the test data [21][22].

Convolution and pooling layers will be repeated many times depending on the CNN desired architecture and finally reach the final layer of the network named, The Fully Connected layer as shown in Figure 1. The features from the previous layers are forward-propagated through the network and fed into this fully connected layer with an output layer. For learning the classifier model, the conventional backpropagation learning algorithm is used to train the fully connected network and update the model parameters via gradient descent update rule [23]

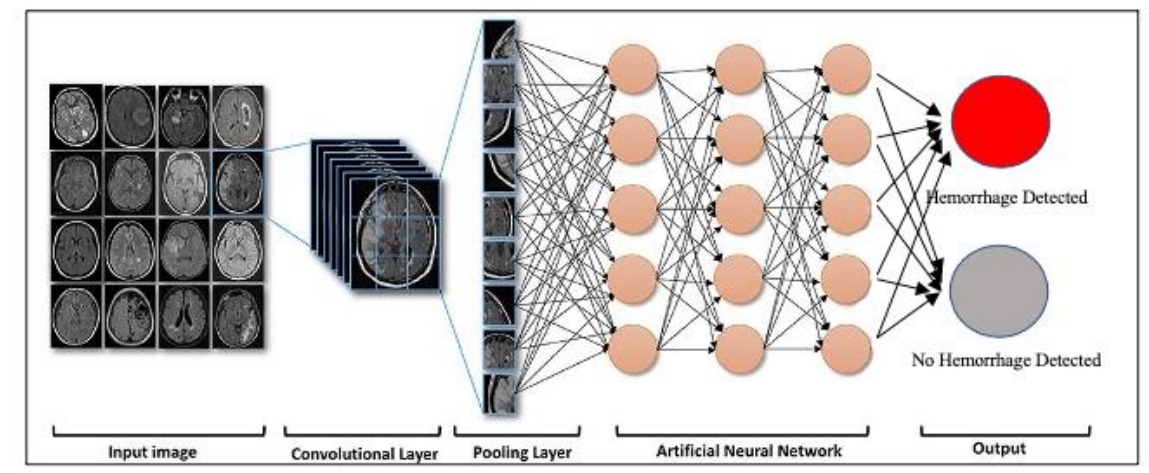

Figure 1. The Concept of Convolutional Neural Network

\subsection{Data Analysis}

To analyze the algorithm model performance, we used a confusion matrix to measure the accuracy, sensitivity, specificity, precision, and F1 score [24]. The confusion matrix is a matrix sized $2 \times 2$ for binary classification with actual values on one axis and predicted on another. We classified the result into a true positive, true negative, false negative, and false-positive as shown in Table 1. The true positive (TP) model correctly predicts the positive class (prediction and actual both are positive). The true negative (TN) model correctly predicts the negative class (prediction and actual both are negative). The false-positive (FP) model gives the wrong prediction of the negative class (predicted-positive, actual-negative). The false-negative (FN) model wrongly predicts the positive class (predicted-negative, actual-positive). 
Table 1. Example of Confusion Matrix

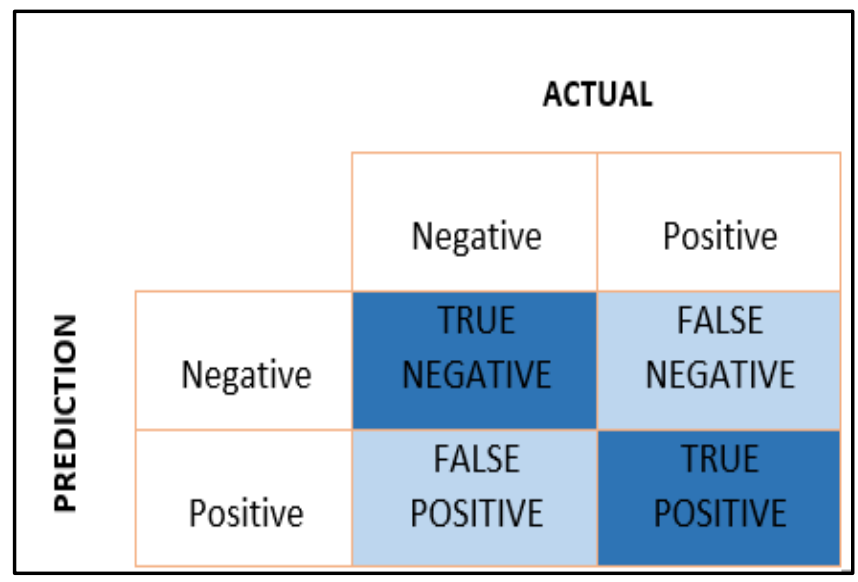

From the confusion matrix, we can measure precision by calculating the percentage of true positive results as shown in Equation 1. We also can measure sensitivity and specificity based on the formula shown in Equation 2 and Equation 3. F1 score is the harmonic mean of the precision and sensitivity as shown in Equation 4. The highest possible value of an F-score is 1, indicating perfect precision and recall, and the lowest possible value is 0 if either the precision or the recall is zero.

$$
\begin{gathered}
\text { Precesion }=T P /(T P+F P) \\
\text { Sensivity }=T P /(T P+F N) \\
\text { Specificity }=T N /(T N /(F P+T N))
\end{gathered}
$$

F1 score $=(2 *($ Precision $*$ Sensitivity $)) /(($ Precision

$$
+ \text { Sensitivity)) }
$$

\subsection{RESULT}

\subsection{Data Pre-processing and Augmentation}

First, we randomly divided our images into training and validation. A total of 200 datasets were used. We further divided it into two folders, one with head CT with hemorrhage, another folder for head CT without hemorrhage. All images will be converted from DICOM format to PNG format which is the better format to be utilized in any machine learning framework [16]. 
In the pre-processing step, the redundant data that are stored within CT images such as files name, dates, and so on, are manually removed to enhance the performance of classification as shown in Figure 2. Augmentation was done to enrich the information of pre-processed images. This will help pre-empt overfitting or memorization of training data and has been shown to increase the accuracy and generalization of CNNs [25]. In the augmentation step, a few techniques of rotation are used as shown in Figure 3.

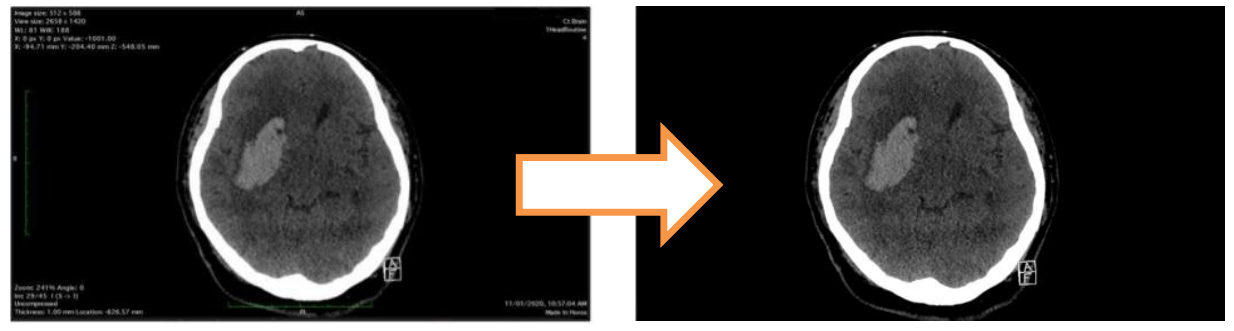

Figure 2. Removing Data from Image Sample

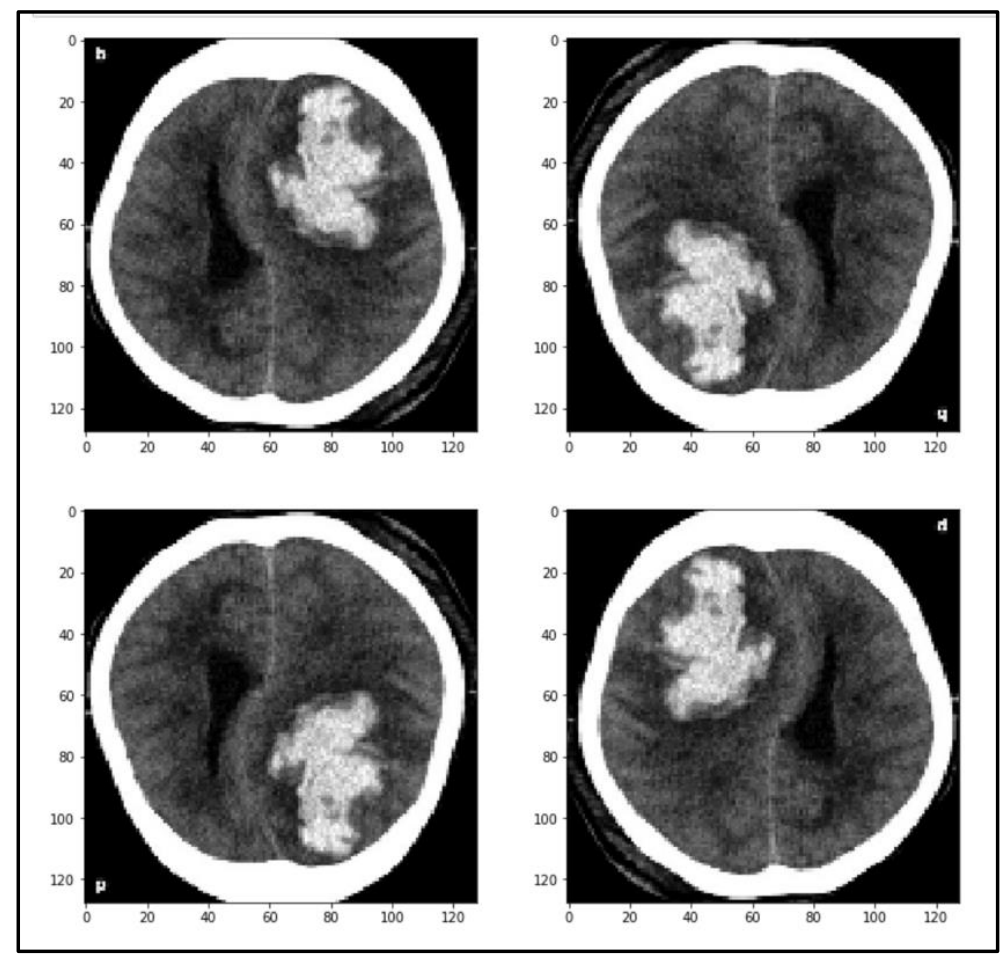

Figure 3. Data is Rotated for the Augmentation Process. Rotated images with 90,180 and 270 degrees. 


\subsection{Convolutional Neural Networks}

Generally, a CNN relies on architectural features which include the receptive field, weight sharing, and pooling operation to take into account the 2D characteristic of structured data such as images [26]. The concept of weight sharing for convolution maps drastically reduces model parameters; this has important implications that the model is less prone to over-fitting as compared to fully connected models of comparable size.

During the training process, the loss and accuracy values are recorded and plotted as shown in Figure 4 . The plot shows that accuracy increases and loss decrease over time during the training process.

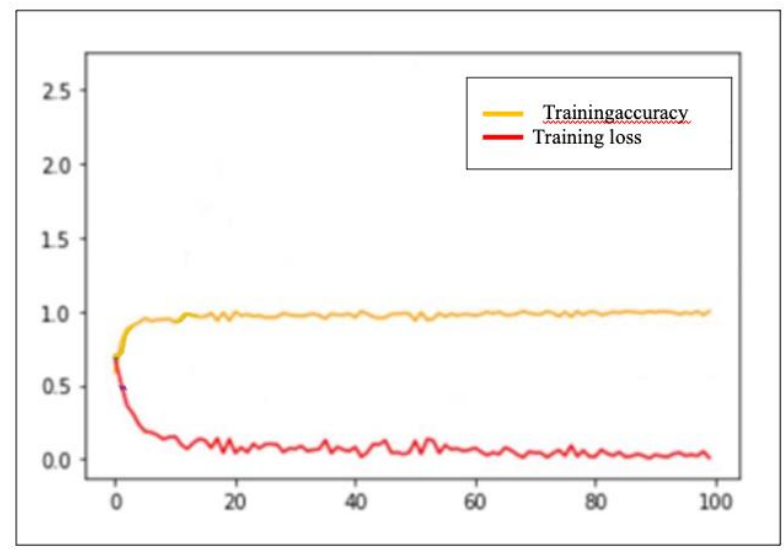

Figure 4. Loss and Accuracy Value during Training and Validation Process

\subsection{Evaluating the Trained Model}

Model performance is evaluated using a confusion matrix to measure the accuracy, precision, sensitivity, specificity, and F1 score. The images to validate the accuracy of the model are shown in Figure 5. Each image is then compared with the list of datasets which was categorized by dataset provider based on CT scan formal report for confirmation of the findings.

The confusion matrix derived from the model showed great performance results. From 200 training data, 95 samples showed true positive, 95 samples showed true negative, 7 samples showed false 
positive, and 3 samples showed false negative as shown in Table 2. From this data, we calculated the sensitivity, specificity, precision, accuracy, and F1 score of the algorithm model. All results showed a great performance score as shown in Table 3. Figure 6 shows one of the examples of intracranial hemorrhage detection using the algorithm model we have created.

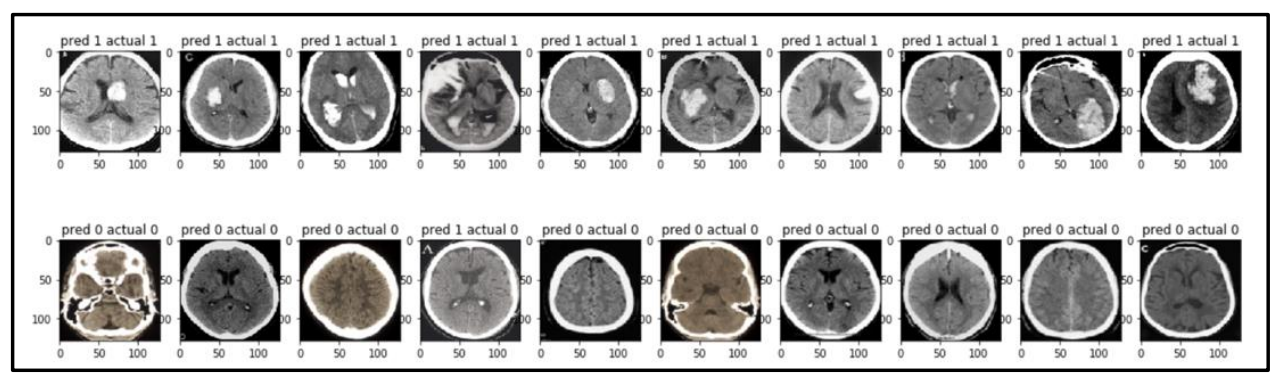

Figure 5. Images Check done during Model Development. Results of $1=$ haemorrhage detected, $0=$ no haemorrhage detected

Table 2. Model Confusion Matrix

\begin{tabular}{|l|c|c|}
\hline & Actual positive & Actual negative \\
\hline Predicted positive & 95 & 7 \\
\hline Predicted negative & 3 & 95 \\
\hline
\end{tabular}

Table 3. The calculation for Sensitivity, Specificity, Precision, Accuracy, and F1 Score derived from Confusion Matrix

\begin{tabular}{|l|c|c|}
\hline Measure & Value & Derivations \\
\hline Sensitivity & 0.9694 & $\mathrm{TPR}=\mathrm{TP} /(\mathrm{TP}+\mathrm{FN})$ \\
\hline Specificity & 0.9314 & $\mathrm{SPC}=\mathrm{TN} /(\mathrm{FP}+\mathrm{TN})$ \\
\hline Precision & 0.9314 & $\mathrm{PPV}=\mathrm{TP} /(\mathrm{TP}+\mathrm{FP})$ \\
\hline Accuracy & 0.9500 & $\mathrm{ACC}=(\mathrm{TP}+\mathrm{TN}) /(\mathrm{P}+\mathrm{N})$ \\
\hline F1 Score & 0.9500 & $\mathrm{~F} 1=2 \mathrm{TP} /(2 \mathrm{TP}+\mathrm{FP}+\mathrm{FN})$ \\
\hline
\end{tabular}




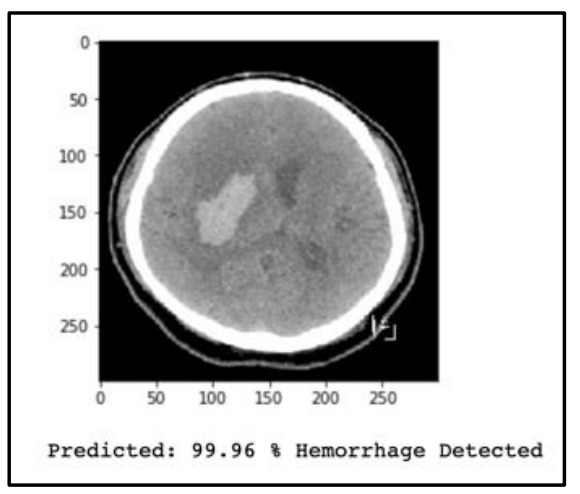

Figure 6. Result of Head CT with Intracranial Hemorrhage Detection

\subsection{DISCUSSION}

Intracranial hemorrhage is one of the common causes of morbidity and mortality in the world [27]. It is a medical emergency and needs to be recognized and treated urgently. Missed diagnosis of intracranial hemorrhage would harm the patient and increase mortality as well as morbidity. Study shows that the mortality rate increased up to $50 \%$ after the hemorrhage within the first 30 days [28]. Medical imaging is an important method to help in making the diagnosis of intracranial hemorrhage. The most common imaging that is used worldwide is a head CT scan. CT scan has high sensitivity and specificity in the detection of hemorrhage especially in acute stages [29].

Over the past decades, the Computer-Aided Diagnosis (CAD) system has been used as an aiding tool to help doctors in making the diagnosis of the head CT scan [30]. It has been shown to help in reducing the workload of doctors [31]. Head CT classification could be a tough task even for a radiologist. This is worst when a lot of head CT scan is involved. Hence, computer-aided diagnosis (CAD) systems have been used to extract useful information from the brain CT to help doctors in having a preliminary quantitative insight about the brain [32]. However, CAD systems can't achieve a highly significant level to make decisions on the type of medical conditions found in a brain CT scan. Thus, the CAD role was left as visualization functionality that helps doctors in making decisions.

Recently, a rapid rising in deep learning was seen. Deep learning has been applied to solve challenging problems in medical fields, such as medical image classification and analysis. It also shows great efficiency in various medical areas such as medical image analysis [33], 
medical organ detection [34], disease detection [35], and many more. In some situations, those algorithms have outperformed the human medical expert's experience in diagnosis. With the help of high network performance and their 'biologically inspired' deep structure, this attracts many researchers to apply deep networks for image classification, including for brain hemorrhage CT image classification. This is because the task to detect hemorrhage could be a tedious task, even for medical experts especially for visualizing the hemorrhage in its early stages.

In this study, 200 samples were used for the training and testing of the algorithm. Deep learning using a convolutional neural network (CNN) has been employed successfully to detect brain hemorrhage. From the result of this algorithm model, intraparenchymal hemorrhages, extradural and subdural hemorrhage was detected with the highest probability. These were typically hyperattenuating and surrounded by normal tissues which make the hemorrhage more obvious compared to other types of intracranial hemorrhage such as subarachnoid hemorrhage and intraventricular hemorrhage. Almost all cases with intraparenchymal, epidural, and subdural hemorrhage gave true positive results. Only one case of subdural hemorrhage gave a false negative result. This is due to its location at the left temporal region likely due to its small size and obscured by beam hardening artifact of adjacent bones as shown in Figure 7.

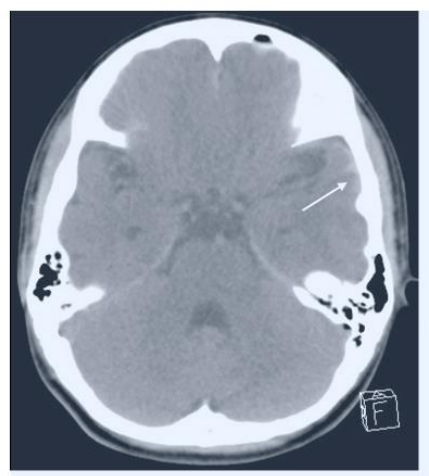

Figure 7. Acute Subdural Hemorrhage at Left Temporal Region Missed Interpreted as Negative Result of Hemorrhage by Algorithm Module

The subarachnoid hemorrhage is relatively difficult to detect especially in a small amount of hemorrhage. This type of hemorrhage is typically narrow with blood filling in the sulci. In some cases, this type of hemorrhage has the same attenuation with brain parenchyma 
which makes the detection even more challenging. Two cases of subarachnoid hemorrhage showed false negative results as shown in Figure 8, the same issue with intraventricular hemorrhage. One case with intraventricular hemorrhage gave false negative result. This is due to the small size and located at posterior of the occipital horn.

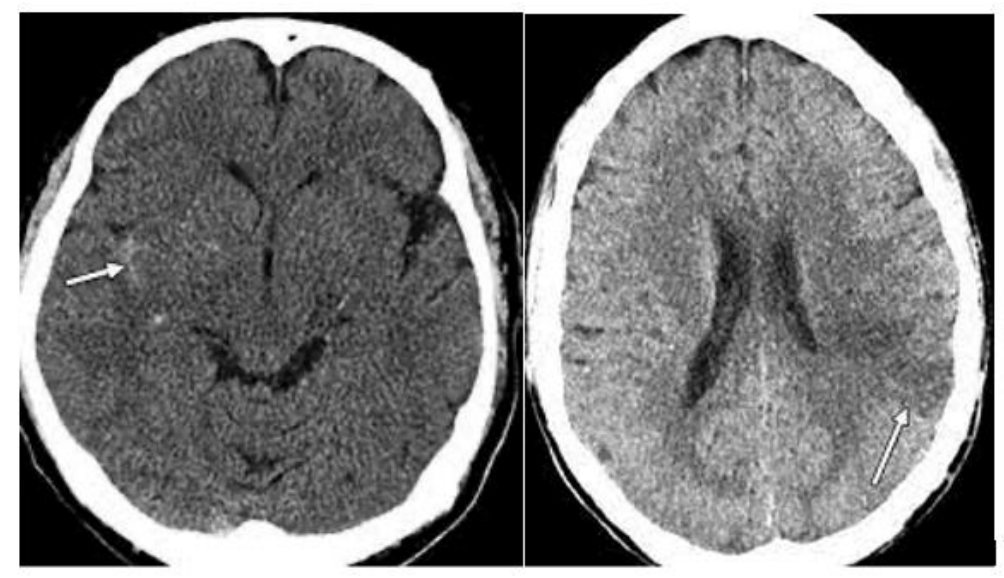

Figure 8. Acute Subarachnoid Hemorrhage (arrow) Missed Interpreted as Negative Hemorrhage by Algorithm Module

There are 7 cases that gave false-positive results. This is due to the presence of calcification in the brain parenchyma. Normal calcification usually has attenuation $>100 \mathrm{HU}$ [36]. But in some cases, the calcification can give attenuation of $50-100 \mathrm{HU}$ which is the same attenuation with hemorrhage which makes it difficult to differentiate with hemorrhage as shown in Figure 9. As a radiologist, they will recognize the typical area of calcification and correlate with the patient clinical findings. This will help them to decide between calcification and hemorrhage in head CT. As for deep learning, the algorithm module will be evaluated solely on the attenuation of lesions in head CT.

The overall sensitivity of this algorithm is $95.9 \%$ which is a great achievement. But some of the cases show false-positive results which cannot be allowed in real medical practice. Moreover, this algorithm module is only able to detect acute hemorrhage. The hyperacute, subacute, and chronic hemorrhage cannot be detected using this algorithm module due to different attenuation of hemorrhage [37]. Hence, this algorithm module is just a supplementary method to help doctors in the early detection of acute intracranial hemorrhage. The need for evaluation by the radiologist is compulsory to form a final 
official report of the head CT.

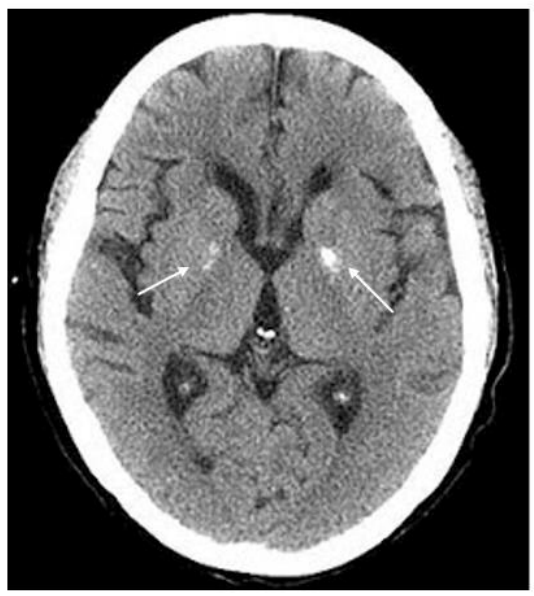

Figure 9. Basal Ganglia Calcification Missed Interpreted as Hemorrhage

\subsection{CONCLUSION}

In this study, we have successfully developed an algorithm model and proved that deep learning by using $\mathrm{CNN}$ enables us to create an accurate classifier that can differentiate between head CT scan with hemorrhage and without hemorrhage. The algorithm model will help to reduce the probability of missed interpretation of intracranial hemorrhage in the head CT scan. This is very crucial as missed findings of intracranial hemorrhage in the head CT caused delayed management to the patient. This will cause significant morbidity and mortality to the patient since "time is brain", increased speed and reduced error in these clinical settings would constitute life-saving innovations.

\subsection{DECLARATION OF COMPETING INTEREST}

The authors declare that they have no known competing financial interests or personal relationships that could have appeared to influence the work reported in this paper. 


\subsection{ACKNOWLEDGMENTS}

I would like to acknowledge everyone who had contributed directly or indirectly to my research accomplishment.

First and foremost, I would like to express my deepest gratitude to my research supervisor, Prof. Dr. Ahmad Bin Sobri who had given me invaluable guidance, encouragement, and continuous supervision throughout my study period. This study would not be possible without his help.

Sincere thanks also go to my research co-supervisor, Dr. Aqilah Binti Baseri Huddin for providing me advice and endless knowledge regarding artificial intelligence. Her interest in artificial intelligence had indeed inspired me.

\subsection{REFERENCES}

[1] K. Blennow et al., "Traumatic brain injuries," Nature Reviews Disease Primers, vol. 2, pp. 1-19, 2016, doi: 10.1038/nrdp.2016.84.

[2] J. Borsch, "Ovulationshemmer oder abtreibungspille? So wirken notfallkontrazeptiva," Deutsche Apotheker Zeitung, vol. 153, no. 9, pp. 32-33, 2013.

[3] A. M. Naidech, "Intracranial hemorrhage," American Journal of Respiratory and Critical Care Medicine, vol. 184, no. 9, pp. 998-1006, 2011, doi: 10.1164/rccm.201103-0475CI.

[4] G. Rath and B. Ray, "Head Injury: Assessment and Early Management," Practice Guidelines in Anesthesia, no. January 2014, pp. 53-53, 2016, doi: 10.5005/jp/books/12644_7.

[5] W. M. Strub, J. L. Leach, T. Tomsick, and A. Vagal, “Overnight preliminary head CT interpretations provided by residents: Locations of misidentified intracranial hemorrhage," American Journal of Neuroradiology, vol. 28, no. 9, pp. 1679-1682, 2007, doi: 10.3174/ajnr.A0653.

[6] J. S. Moon et al., "Prehospital neurologic deterioration in patients with intracerebral hemorrhage," Critical Care Medicine, vol. 36, no. 1, pp. 172-175, 2008, doi: 10.1097/01.CCM.0000297876.62464.6B.

[7] J. C. Hemphill et al., "Guidelines for the Management of Spontaneous Intracerebral Hemorrhage: A Guideline for Healthcare Professionals from the American Heart 
Association/American Stroke Association," Stroke, vol. 46, no. 7, pp. 2032-2060, 2015, doi: 10.1161/STR.0000000000000069.

[8] B. E. Bejnordi et al., "Diagnostic assessment of deep learning algorithms for detection of lymph node metastases in women with breast cancer," JAMA - Journal of the American Medical Association, vol. 318, no. 22, pp. 2199-2210, 2017, doi: 10.1001/jama.2017.14585.

[9] A. Esteva et al., "Dermatologist-level classification of skin cancer with deep neural networks," Nature, vol. 542, no. 7639, pp. 115118, 2017, doi: 10.1038/nature21056.

[10] V. Gulshan et al., "Development and validation of a deep learning algorithm for detection of diabetic retinopathy in retinal fundus photographs," JAMA - Journal of the American Medical Association, vol. 316, no. 22, pp. 2402-2410, 2016, doi: 10.1001/jama.2016.17216.

[11] Y. Lecun, Y. Bengio, and G. Hinton, “Deep learning," Nature, vol. 521, no. 7553, pp. 436-444, 2015, doi: 10.1038/nature14539.

[12] C. Tang, Q. Zhu, W. Wu, W. Huang, C. Hong, and X. Niu, "PLANET: Improved Convolutional Neural Networks with Image Enhancement for Image Classification," Mathematical Problems in Engineering, vol. 2020, 2020, doi: 10.1155/2020/1245924.

[13] A. Usha Ruby, P. Theerthagiri, I. Jeena Jacob, and Y. Vamsidhar, "Binary cross entropy with deep learning technique for image classification," International Journal of Advanced Trends in Computer Science and Engineering, vol. 9, no. 4, pp. 5393-5397, 2020, doi: 10.30534/ijatcse/2020/175942020.

[14] A. Pinto, "Spectrum of diagnostic errors in radiology," World Journal of Radiology, vol. 2, no. 10, p. 377, 2010, doi: 10.4329/wjr.v2.i10.377.

[15] A. Helwan, G. El-Fakhri, H. Sasani, and D. Uzun Ozsahin, "Deep networks in identifying CT brain hemorrhage," Journal of Intelligent and Fuzzy Systems, vol. 35, no. 2, pp. 2215-2228, 2018, doi: 10.3233/JIFS-172261.

[16] P. Lakhani, D. L. Gray, C. R. Pett, P. Nagy, and G. Shih, "Hello World Deep Learning in Medical Imaging," 2018.

[17] А. Якимчик, “Jupyter Notebook: система интерактивных научных вычислений," 2019.

[18] R. Browse and J. Glasgow, "Programming Artificial Languages 
for," vol. 11, pp. 431-448, 1984.

[19] M. Matsugu, K. Mori, Y. Mitari, and Y. Kaneda, "Subject independent facial expression recognition with robust face detection using a convolutional neural network," Neural Networks, vol. 16, no. 5-6, pp. 555-559, 2003, doi: 10.1016/S08936080(03)00115-1.

[20] P. Baldi, "Autoencoders, Unsupervised Learning, and Deep Architectures," ICML Unsupervised and Transfer Learning, pp. 3750, 2012, doi: 10.1561/2200000006.

[21] K. Simonyan and A. Zisserman, "Very deep convolutional networks for large-scale image recognition," 3rd International Conference on Learning Representations, ICLR 2015 - Conference Track Proceedings, pp. 1-14, 2015.

[22] J. Weston, F. Ratle, and R. Collobert, "Deep learning via semisupervised embedding," Proceedings of the 25th International Conference on Machine Learning, pp. 1168-1175, 2008, doi: 10.1145/1390156.1390303.

[23] D. Erhan, A. Courville, Y. Bengio, and P. Vincent, "Why does unsupervised pre-training help deep learning?," Journal of Machine Learning Research, vol. 9, pp. 201-208, 2010.

[24] D. Visa Sofia, "Confusion Matrix-based Feature Selection Sofia Visa," ConfusionMatrix-based Feature Selection Sofia, vol. 710, no. January, p. 8, 2011.

[25] R. Wu, S. Yan, Y. Shan, Q. Dang, and G. Sun, “Deep Image: Scaling up Image Recognition," 2015.

[26] T. F. Gonzalez, "Handbook of approximation algorithms and metaheuristics," Handbook of Approximation Algorithms and Metaheuristics, pp. 1-1432, 2007, doi: 10.1201/9781420010749.

[27] U. Balasooriya and M. U. S. Perera, "Intelligent brain hemorrhage diagnosis using artificial neural networks," BEIAC 2012 - 2012 IEEE Business, Engineering and Industrial Applications Colloquium, pp. 128-133, 2012, doi: 10.1109/BEIAC.2012.6226036.

[28] R. Badenes and F. Bilotta, "Neurocritical care for intracranial haemorrhage: A systematic review of recent studies," British Journal of Anaesthesia, vol. 115, no. December, pp. ii68-ii74, 2015, doi: 10.1093/bja/aev379.

[29] F. Alobeidi and R. I. Aviv, "Basel Emergency Imaging of 
Intracerebral Haemorrhage," Frontiers of Neurology and Neuroscience, vol. 37, pp. 13-26, 2015, doi: 10.1159/000437110.

[30] J. Napier, C. J. Debono, P. Bezzina, and F. Zarb, "A CAD System for Brain Haemorrhage Detection in Head CT Scans," EUROCON 2019 - 18th International Conference on Smart Technologies, pp. 1-6, 2019, doi: 10.1109/EUROCON.2019.8861833.

[31] Q. Li and R. M. Nishikawa, "Computer-aided detection and diagnosis in medical imaging," Computer-Aided Detection and Diagnosis in Medical Imaging, pp. 1-425, 2015, doi: 10.1201/b18191.

[32] S. Yu and L. Guan, "A CAD system for the automatic detection of clustered microcalcifications in digitized mammogram films," IEEE Transactions on Medical Imaging, vol. 19, no. 2, pp. 115-126, 2000, doi: 10.1109/42.836371.

[33] D. Hutchison, "and Data Labeling," vol. 2, pp. 21-29, 2016, doi: 10.1007/978-3-319-46976-8.

[34] A. Helwan and D. Uzun Ozsahin, "Sliding Window Based Machine Learning System for the Left Ventricle Localization in MR Cardiac Images," Applied Computational Intelligence and Soft Computing, vol. 2017, 2017, doi: 10.1155/2017/3048181.

[35] S. Albarqouni, C. Baur, F. Achilles, V. Belagiannis, S. Demirci, and N. Navab, "AggNet: Deep Learning From Crowds for Mitosis Detection in Breast Cancer Histology Images," IEEE Transactions on Medical Imaging, vol. 35, no. 5, pp. 1313-1321, 2016, doi: 10.1109/TMI.2016.2528120.

[36] J. L. Nute, L. Le Roux, A. G. Chandler, V. Baladandayuthapani, D. Schellingerhout, and D. D. Cody, "Differentiation of lowattenuation intracranial hemorrhage and calcification using dualenergy computed tomography in a phantom system," Investigative Radiology, vol. 50, no. 1, pp. 9-16, 2015, doi: 10.1097/RLI.0000000000000089.

[37] P. M. Parizel, S. Makkat, E. Van Miert, J. W. Van Goethem, L. Van den Hauwe, and A. M. De Schepper, "Intracranial hemorrhage: Principles of CT and MRI interpretation," European Radiology, vol. 11, no. 9, pp. 1770-1783, 2001, doi: 10.1007/s003300000800. 